\title{
Olfactory-based social learning varies as a function of parity in female rats
}

\author{
A. S. FLEMING, C. KUCHERA, and A. LEE \\ University of Toronto, Erindale College, Mississauga, Ontario, Canada \\ and \\ G. WINOCUR \\ Rotman Research Institute, Baycrest Hospital, Toronto, Ontario, Canada
}

\begin{abstract}
New mother rats, under the influence of parturitional hormones, are nurturant as soon as their pups are born. Maternal experiences acquired under the influence of these hormones are retained for a considerable period beyond parturition. This effect is more robust in the postpartum animal than in a nulliparous animal that has been induced to become maternal nonhormonally through daily exposure to pups. The present experiments were designed to determine whether the parity difference in robustness of a maternal experience reflects a parity difference in the ease of acquiring any task, or whether the learning must be specific to pups and the maternal context. In the first experiment, primiparous and nulliparous female rats were compared in their performance on a socially conditioned food preference task. Each animal was exposed to a conspecific that had previously eaten a new food and was then tested for amount consumed of the preexposed as opposed to nonpreexposed new food. The animals were exposed to their conspecifics for 5,15 , or $30 \mathrm{~min}$ and were tested 7 days later. Both parity groups and all three exposure groups exhibited a significant preference for the preexposed diet, but a higher proportion of the postpartum animals did so. In the second experiment, postpartum and nulliparous animals were compared in their ability to recognize juvenile animals to whom they had been preexposed. Exposures lasted $30 \mathrm{~min}$ and exposure test intervals were 1, 3, or 5 days. Again, although both groups recognized the juveniles, the postpartum group showed a stronger recognition effect (reflected in change in investigation scores from exposure to reexposure). Thus, although the effects found were small, both experiments indicate that parity influences the robustness of social learning in a direction favoring the postpartum animal.
\end{abstract}

If newly parturient mother rats are separated from their litters at the time of parturition and are tested for their maternal responsiveness 10 days later, they are no more responsive than inexperienced nulliparous animals; however, if dams are permitted to interact with their litters for as little as $1 \mathrm{~h}$ at the time of parturition (Fleming \& Sarker, 1990) or the day after (Malenfant, Barry, \& Fleming, 1991), but prior to separation, at retention testing on Day 10 postpartum, they either are immediately maternal or become maternal with very little pup exposure (Fleming \& Sarker, 1990; Malenfant et al., 1991). This long-term retention of maternal responsiveness we have termed the maternal experience effect.

In order to determine whether newly parturient animals are uniquely primed to benefit from a brief maternal experience, maternal dams and maternal virgins, induced to become maternal through pup induction procedures

This research was supported by a N.S.E.R.C. operating grant to A.S.F. Many thanks to Lisa Cauchi for her help with the manuscript. Correspondence should be addressed to A. S. Fleming, Department of Psychology, University of Toronto, Erindale College, Mississauga, ON, Canada L5L 1C6 (e-mail: afleming@credit.erin or fleming@psych. utoronto.ca).
(Fleming \& Sarker, 1990), were given a 1-day interactive experience with pups and tested for their maternal responsiveness 30 days later. In comparison with dams, previously maternal virgins took considerably longer to reinitiate maternal behavior, a difference that was further accentuated if retention testing occurred under conditions of hormonal priming, during a subsequent pregnancy or following exogenous hormone treatment (Fleming \& Sarker, 1990). Moreover, we have evidence that during the interactive experience dams are receiving somatosensory and olfactory stimulation and both modalities are activated in the long-term retention of the behavior (Malenfant et al., 1991; Morgan, Fleming, \& Stern, 1991).

How the parturitional state facilitates the maternal experience effect is not known. It is possible that the associated hormonal environment facilitates all kinds of learning independently of the learning context, and that the rapid maternal learning is just an instance of a more general facilitation or capacity. Alternatively, it could be that the periparturitional hormones simply heighten the salience of rat pups for the dam and that it is easier for the dam to acquire and retain experiences if the pups have salience than if they do not. In the present paper, we investigate the first of these two hypotheses, by comparing 
the performance of nulliparous and parturient females on two species-characteristic tasks where olfaction and somatosensory stimulation are important modalities. In a second paper (Fleming, Korsmit, \& Deller, 1994), we explore the pup salience hypothesis.

In the present study, we used two different learning tasks, which resembled the maternal experience insofar as learning occurred during a simple brief exposure period, involved a social interaction and associated tactile and olfactory features, and could be retained for up to a week or more. The first, a socially conditioned food preference task, has been used extensively by Galef and his colleagues (Galef, 1989; Galef, Kennett, \& Stein, 1985; Galef \& Stein, 1985; Galef \& Wigmore, 1983). Pairs of animals that have been cohabiting are separated, and one of the pair (the demonstrator) is given a novel food to eat. The demonstrator is then returned to the home cage and allowed to interact with the observer for variable periods of time (e.g., 2 min to $2 \mathrm{~h}$ ). One to 8 days later, the observer is provided with two novel foods-the food given to the demonstrator, and an alternative choice. Using the proportional measure (demonstrator food/ demonstrator + alternative food intake) as a measure of the food preference, Galef found that the observer showed a preference for the food previously associated with the demonstrator as long as the observer was permitted to interact with the demonstrator or, at the least, nosed and sniffed around the demonstrator's mouth region.

The second task involves a socially mediated recognition paradigm in which an adult animal is permitted to briefly interact with a juvenile and at retention testing is presented either with the same juvenile or with a different juvenile. The adult exhibits individual recognition if it shows a greater reduction in investigatory behavior in the presence of the same, than in the presence of the novel, juvenile (Thor \& Holloway, 1982). Again social interactions seem to mediate this form of learning (Sawyer, Hengehold, \& Perez, 1984; Thor \& Holloway, 1982). Since there is no reason to believe that either novel foods or postweaning juveniles have particular salience for postpartum dams, any evidence of more efficient or robust learning on the part of dams, in comparison with virgins, would reflect relative differences in their learning capacity. Alternatively, no differences in their performance on these tasks would suggest that the heightened maternal experience effect shown by dams is due to differences between the two kinds of animal in their perception of, or reaction to, the pup stimulus.

\section{EXPERIMENT 1 \\ Conditioned Food Preferences in Postpartum and Virgin Females}

In this experiment, groups of newly parturient dams or nulliparous virgins were tested on the conditioned food preference task, with a variety of exposure durations and an 8-day exposure-test interval. The exposure intervals were varied to ensure that the task would be adequately difficult to reveal parity differences should these exist.

\section{Method}

\section{Subjects and Housing}

Subjects were 60 - to 90 -day-old virgin female rats born at Erindale College, University of Toronto from a stock originally obtained from Charles Rivers Farms in Quebec. The animals were maintained under a 12:12-h light:dark cycle, with lights on at $0800 \mathrm{~h}$. Room temperature and humidity were maintained at $24.0^{\circ} \mathrm{C}$ and $40 \%$, respectively.

\section{Experimental Diets}

The cocoa diet (COC) consisted of powdered Purina Lab Chow mixed with unsweetened Hershey's cocoa ( $2 \%$ by weight) and the cinnamon diet (CIN) consisted of powdered Purina Lab Chow and Club House ground cinnamon ( $1 \%$ by weight). The diets were mixed 1 day before they were needed and refrigerated to ensure uniform freshness throughout the experiment.

\section{Procedure}

Groups. One hundred and thirty-one rats were divided into groups of observer animals. The observers were divided into matched postpartum (PP) and virgin (V) groups and randomly assigned to one of three exposure groups: 5,15 , or $30 \mathrm{~min}$. Within each exposure condition, animals were exposed to either COC or CIN. During preexposure, the observers were paired with one of 65 demonstrator animals that were 60-90 days old at the beginning of the experiment and had been randomly assigned to a specific diet type. The following 12 groups were formed: PPCOC5 $(n=9)$, PPCOC15 $(n=11)$, or PPCOC30 $(n=10)$; PPCIN5 $(n=10)$, PPCIN15 $(n=21)$, or PPCIN30 $(n=10)$; VCOC5 $(n=10)$, VCOC15 $(n=10)$, or $\operatorname{VCOC} 30(n=10)$; and VCIN5 $(n=10)$, VCIN15 $(n=10)$, or VCIN30 $(n=10)$.

The procedure was divided into the following three phases.

Preexposure. Rats were housed individually in standard polypropylene shoebox cages $(48.3 \times 26.7 \times 15.6 \mathrm{~cm})$. Observers in the PP groups were mated by being placed into the cage of a sexually active male for a 5-day period. Twenty-one days after mating (Day 1 with male), females were transferred to the test cage (polycarbonate cages, $45.9 \times 45.9 \times 29 \mathrm{~cm}$ ), where they were placed with a nonpregnant demonstrator. The matched $\mathrm{V}$ groups were also placed with demonstrators at the same time. PP females were allowed to give birth in the test cage in the presence of the demonstrators. If the PP observers did not give birth within 3 days, they and their matched $\mathrm{V}$ animals were removed from the study.

PP animals were checked for the pups two times daily, at 0900 and $1700 \mathrm{~h}$. When parturition occurred, the pups were removed and the associated demonstrator was moved to another cage. If females were giving birth during a spot check, the demonstrator was removed but the pups were left with the dam until the next spot check.

Exposure. The exposure phase occurred at $0900 \mathrm{~h}$, either 16 or $24 \mathrm{~h}$ after parturition, depending on whether pups were found the previous 1700 or $0900 \mathrm{~h}$, respectively.

Prior to exposure, the demonstrators were food deprived for $23 \mathrm{~h}$, removed to a room away from their respective observer and fed either COC or CIN for $60 \mathrm{~min}$. In addition, each demonstrator's snout was dusted with the diet three times immediately before being placed into the test cage with the associated observer. Depending on the group, the demonstrator remained with the observer for 5 , 15 , or $30 \mathrm{~min}$. Both rats were then returned to their home cages, where Purina rat chow and water were available ad lib.

The $V$ animals were exposed to their demonstrators when they were in either the metestrus or diestrous phase of their estrous cycles. On the PP animals' exposure day, the matched V females were smeared to determine stage of the vaginal cycle. If animals were in proestrus or estrus-indicating recently elevated estradiol levelstheir exposure phases were delayed until metestrus or diestrus.

Testing. Seven days after exposure, all observers were food deprived for $23 \mathrm{~h}$. On Day 8, observers were placed into the test cage and offered two food cups, each containing $25 \mathrm{~g}$ of food which 
was either CIN or COC flavored. Food cups were positioned at the front left and right corners of the cage and counterbalanced across animals. The food cups were weighed (to the nearest $0.1 \mathrm{~g}$ ) before and after the 2 -h consumption period. To remove debris, fecal pellets, or other matter from the food cups, food was strained twice through a sieve prior to weighing.

\section{Results}

To determine whether there were differences in conditioned food preference as a function of the exposure interval and preexposed diet, for each group separately, a 3 (duration: 5,15 , or $30 \mathrm{~min}$ ) $\times 2$ (preexposed diet: CIN vs. COC) analysis of variance (ANOVA) was performed on the proportion of total diet consumed as the preexposed diet as well as on the total food intake.

For neither parity condition was there a significant difference in proportional food intake as a function of exposure duration, diet type, or the interaction of these factors. Moreover, collapsing across diets and exposure conditions, a subsequent one-way ANOVA to compare the virgin and postpartum animals showed that postpartum animals ingested a significantly higher proportion of their intake as the preexposed diet than did the virgin animals $[F(1,141)=4.3, p<.04]$. (See Figure 1.)

Both parity groups (collapsing across exposure) and all three exposure groups (collapsing across parity conditions) exhibited significant preferences for the preexposed diet (parity: virgins, $69 \%$; binomial test, $p<.001$; postpartum, $81 \%$, binomial, $p<.000$; exposure: $5 \mathrm{~min}, 82 \%$, binomial, $p<.01 ; 15 \mathrm{~min}, 73 \%$, binomial, $p<.000$; and $30 \mathrm{~min}, 82 \%$, binomial, $p<.000$ ). However, a chisquare analysis of the two parity groups, controlling for exposure condition, showed a significant effect $\left[\chi^{2}(1)=\right.$ $3.92, p<.047$ ]; as shown in Figure 2, a higher proportion of the postpartum than of the virgin animals exhibited a food preference.

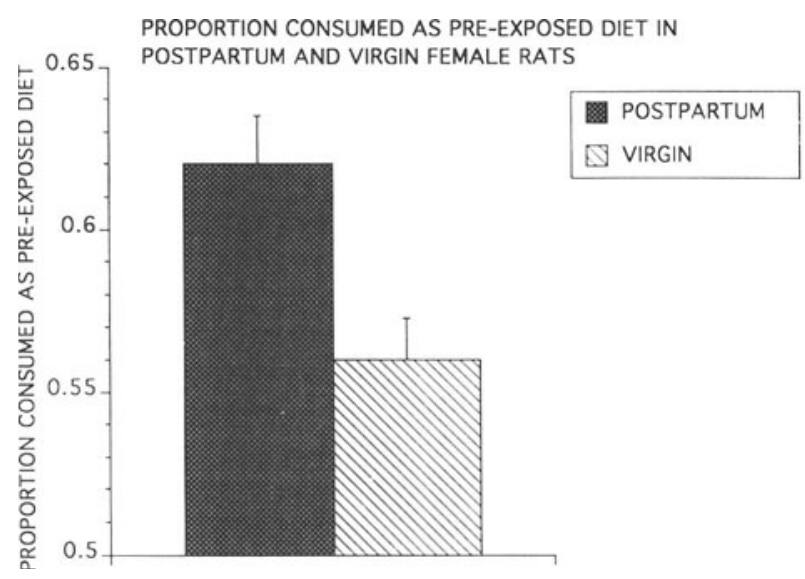

Figure 1. Proportion of total diet consumed as the preexposed diet in postpartum and virgin female rats $(\bar{X} \pm S E M)$.

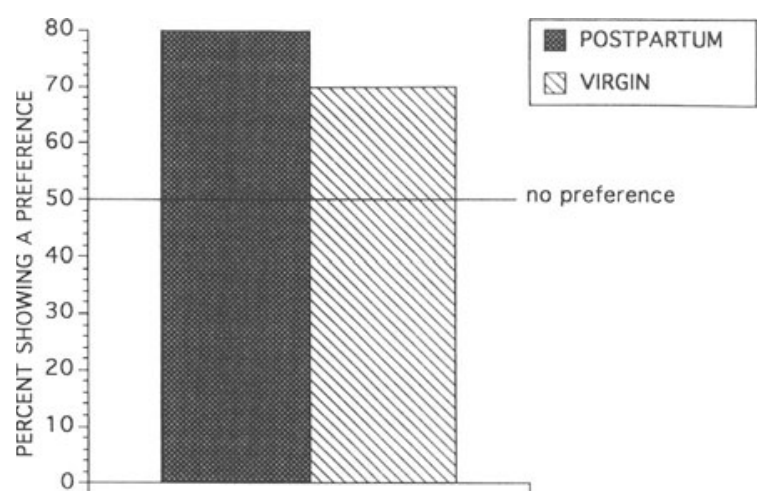

Figure 2. Percent of animals in each parity condition showing a preference for the preexposed diet.

Results of the two-way ANOVAS for each parity group on the total diet eaten as a function of diet (CIN vs. COC) and exposure duration $(5,15$, and $30 \mathrm{~min})$ indicated no significant main effects or interactions. Similarly, one-way ANOVAS to compare the two parity conditions showed no effects.

\section{Discussion}

These data show that PP animals are somewhat superior to $\mathrm{V}$ animals in the development of a conditioned food preference, using socially mediated cues. The fact that there were no parity differences in the total amount of food consumed during the test period indicates that parity differences in hunger motivation probably does not mediate the preference differences. Also, although the PP group did engage in significantly more exploration of the conspecific during the exposure phase, the absence of a significant relation between performance during exposure and preference during test suggests that the behavioral differences between PP and V animals do not mediate the preference differences. Regardless of parity, animals are able to learn the food preference task with very little exposure to the food-adulterated conspecific; in fact, with as little as $5 \mathrm{~min}$ of exposure to the new food, animals develop and retain a preference for the food up to 8 days later. According to earlier work by Galef and Stein (1985), this preference depends on exposure to the gaseous breath of a familiar conspecific who has ingested the new food, suggesting that olfactory stimulation is probably important to the effect. We also have evidence that the learning involved in the development of a preference is similar to other types of learning, in that it can be blocked by hippocampal lesions (Winocur, 1990) and by antagonists to $N$-methyl-D-aspartate, a neuropeptide known to be implicated in the acquisition of other memory tasks (Myhal \& Fleming, 1990). Taken together, these results support the idea that the new mother rat shows a heightened ability to learn new tasks and that these tasks need not be directly related to pups and the maternal context. 


\section{EXPERIMENT 2 Social Recognition Learning in Postpartum and Virgin Female Rats}

To establish the generalizability of the facilitated learning exhibited by PP dams, in Experiment 2 we asked whether new mother rats also learn a social recognition task more readily than do virgin counterparts. We used the same basic temporal parameters as those in Experiment 1. PP animals were exposed to a juvenile on the day after birth for a 30 -min period and tested for recognition with either the same or a different juvenile after varying intervals. This study differed from the first in that the tobe-remembered stimulus was a juvenile, not a food; and in this case, the exposure-test interval was varied, not the exposure duration.

\section{Method \\ Subjects, Housing, and Maintenance \\ The animals were 60-90 days of age, derived from the same breeding stock as those in Experiment 1 and maintained under the same conditions. Juvenile male rats (20-25 days of age) served as stimulus animals. They were housed under the same conditions as were the experimental animals, but in a separate room.}

\section{Procedure}

One hundred and twenty-seven experimental rats were used in this study: $63 \mathrm{PP}$ and $64 \mathrm{~V}$ females. Within each of these parity groups, animals were tested 1,3 , or 5 days after a 30-min exposure to a male juvenile (20-25 days old); at test, the juveniles were either the same as, or different from, the juveniles used at exposure. Thus, the following groups were formed: postpartum-same $(\mathrm{PP} / \mathrm{S}$ ), tested 1,3 , or 5 days after exposure (Groups $\mathrm{PP} / \mathrm{S} / 1$, $n=11 ; \mathrm{PP} / \mathrm{S} / 3, n=9$; and $\mathrm{PP} / \mathrm{S} / 5, n=13$ ); postpartum-different (PP/D), tested 1,3 , or 5 days after exposure (Groups PP/D/1, $n=10 ; \mathrm{PP} / \mathrm{D} / 3, n=9 ;$ and $\mathrm{PP} / \mathrm{D} / 5, n=10$ ); virgin-same $(\mathrm{V} / \mathrm{S})$, tested 1,3 , or 5 days after exposure (Groups V/S $/ 1, n=11 ; \mathrm{V} / \mathrm{S} / 3$, $n=10$; and $\mathrm{V} / \mathrm{S} / 5, n=13)$; and virgin-different (V/D), tested 1,3 , or 5 days after exposure (Groups $\mathrm{V} / \mathrm{D} / 1, n=10 ; \mathrm{V} / \mathrm{D} / 3$, $n=10$; and $\mathrm{V} / \mathrm{D} / 5, n=10$ ).

Rats were housed individually in standard polypropylene shoebox cages $(48.3 \times 26.7 \times 15.6 \mathrm{~cm})$. The animals in the PP groups were mated by being placed into the cage of a sexually active male for a 5-day period. Twenty-one days after mating (Day 1 with the male), the females were transferred to large polycarbonate observation cages $(45.9 \times 45.9 \times 29 \mathrm{~cm})$. The matched $V$ groups were placed in large cages at the same time. To check for parturition in PP animals, on Days 21-25 after the 1st day of mating PP animals were checked for the pups two times daily, at 0900 and $1700 \mathrm{~h}$. When parturition occurred, the pups were removed. If females were pupping during a spot check, the pups were left with the dam until the next spot check.

Exposure. The exposure phase occurred at $0900 \mathrm{~h}$, either 16 or $24 \mathrm{~h}$ after parturition, depending on whether pups were found the previous 1700 or $0900 \mathrm{~h}$, respectively.

During the exposure, a 20 - to 25 -day-old male juvenile was placed into the female's cage for a 30 min period. The behavior of the pair of animals was observed and recorded for the first $5 \mathrm{~min}$ of exposure, using an S\&K computer-based event recorder. After the observation both rats were returned to the smaller shoebox cages until the day before test.

Virgin animals were exposed to juveniles when they were in either the metestrus or the diestrus phase of their estrus cycles. On the
PP animals' exposure day, the matched V females were smeared to determine stage of the vaginal cycle. If animals were in proestrus or estrus-indicating recently elevated estradiol levels - their exposure phases were delayed until metestrus or diestrus.

Testing. One, 3 , or 5 days after exposure, all females were tested with either the same juvenile that they encountered during exposure or a different juvenile of the same age. One day prior to test, all animals were returned to the large observation cages. Tests were conducted in the same manner as the original exposure. Juveniles were placed into the females' cages and observations were undertaken for the next $5 \mathrm{~min}$.

\section{Behavioral Observations}

Five-minute observations were made during both the exposure and the test phases using the S\&K computer-based event recorder, which permitted continuous recording of all behaviors simultaneously, with .02-msec intervals. The behavior of the experimental animals was the focus of the observations; the juveniles' behavior was recorded only if it was directed at the experimental animal: The primary behavior that was recorded consisted of sniffing and nosing the juvenile's body; together these two behaviors were called social investigation.

\section{Results}

To determine whether animals within each of the parity groups responded differentially to the juvenile at test as a function of the familiarity dimension (i.e., whether the juvenile was the same or different), ANOVAs were performed in which investigation at exposure and investigation at test constituted the repeated measure. In addition, for planned comparisons, a difference score between investigation at exposure and at test [investigation (exposure) - investigation (test)] was computed. A positive difference score indicates that at test there was less social investigation than during exposure; a negative score indicates the opposite; no difference indicates that behavior at exposure and at test was the same. We predicted that if animals retain a memory for the first juvenile and are presented with a different juvenile at test they should have a smaller difference score on this juvenile-directed behavior than animals presented with the same juvenile. Moreover, if PP animals exhibit a better memory than do $\mathrm{V}$ animals, they should exhibit greater difference scores in relation to the same individuals but not in relation to different individuals. These specific unidirectional predictions were the basis of the planned contrasts and onetailed $p$ values.

To determine whether there were parity differences as a function of the interval between exposure and test, the first set of analyses included three factors (interval, parity, and familiarity) on investigation at each of the two time points. These analyses yielded no significant interval differences.

Since there were no differences as a function of interval, all the different interval groups were combined within each parity-familiarity dimension and a second 4 (groups: $\mathrm{PP} / \mathrm{S}, \mathrm{PP} / \mathrm{D}, \mathrm{V} / \mathrm{S}$, and V/D) $\times 2$ (phase: exposure vs. test) two-way ANOVA was performed with repeated measures on the phase dimension. This showed a significant main effect of phase $[F(1,122)=144, p<.001]$ and 


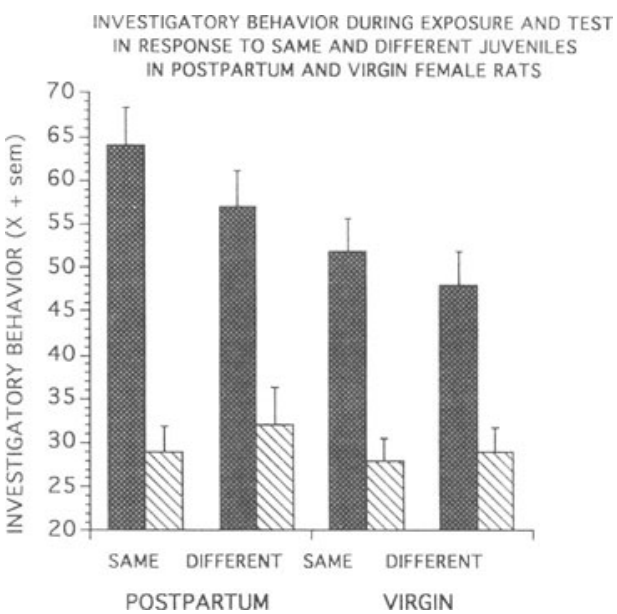

Figure 3. Time spent investigating familiar (same) and unfamiliar (different) juveniles by postpartum and virgin female rats $(\overline{\boldsymbol{X}} \pm \boldsymbol{S E M})$.

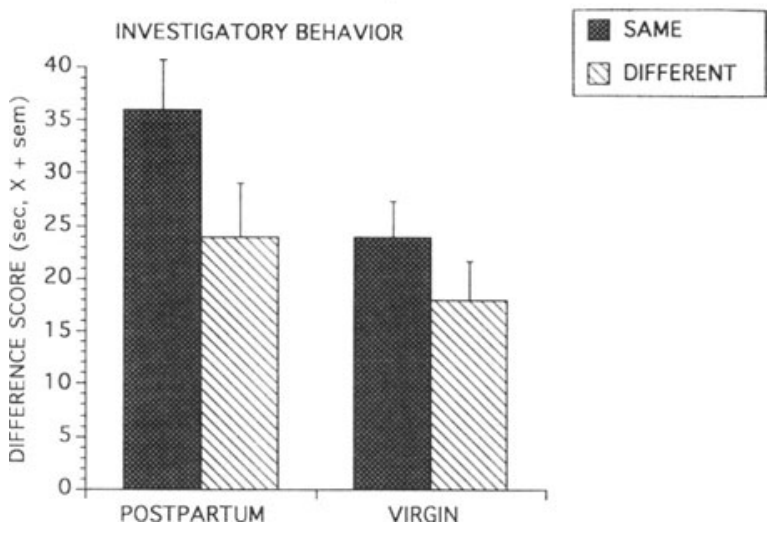

Figure 4. Difference in time spent investigating familiar (same) and unfamiliar (different) juveniles between first and second exposures in postpartum and virgin animals.

a significant group $\times$ phase interaction $[F(3,122)=2.95$, $p<.03$ ]. (See Figure 3.)

As shown in Figure 4, post hoc contrasts comparing difference scores in investigation (exposure - test) indicated that PP/S animals exhibited a marginally bigger reduction in investigation from exposure to test than did group $\mathrm{PP} / \mathrm{D}[t(60)=-1.6, p<.06]$, whereas no such difference was apparent between the two $\mathrm{V}$ groups. Moreover, comparisons between the two parity groups exposed to the same juvenile (PP/S vs. V/S) showed that the PP/S group experienced the bigger reduction in investigation across the two time points $[t(65)=1.9, p<.025]$; comparisons of the two parity groups exposed to a different juvenile (PP/D vs. V/D) showed no differences.

\section{Discussion}

This study, like the first, shows a small but statistically significant advantage among the PP animals in their abil- ity to recognize conspecifics. The absolute difference between the PP and V females may underestimate the size of the effect. Several factors mitigated against the adult rats' recognition of conspecifics. For example, juveniles underwent rapid growth over the several days that elapsed between exposure and test in the present study. During this period, they changed considerably and, consequently, had very different characteristics than they did at original exposure.

Another important observation is that, at test, the stimulus juveniles were always investigated less than at exposure, regardless of parity and familiarity conditions. This may reflect a general tendency to sniff older juveniles less than younger ones and/or a familiarity effect of the procedures that resulted in animals having less curiosity and investigatory drive at test. Despite these methodological complications, significant parity differences in social recognition were evident.

\section{GENERAL DISCUSSION}

Taken together, these experiments indicate that postpartum animals do seem to show more robust learning (or memory) than do nulliparous animals on social learning tasks that do not involve pups or other maternally related cues. Thus, differences in the retention of maternal responsiveness between $\mathrm{PP}$ and $\mathrm{V}$ animals given the same maternal experience (Fleming \& Sarker, 1990) may, in part, reflect a more general enhanced learning capacity in the PP animals. However, the fact that, for both tasks investigated here, the parity effects were quite small indicates that a generally enhanced learning probably does not entirely explain the parity differences seen in the maternal experience effect.

Although the postpartum advantage may be small, the fact that an effect was found in two quite different social learning tasks suggests that the effect is real. How it is mediated, however, is not clear. Given our previous maternal experience studies, it is likely that the parturitional hormones facilitate some aspect of acquisition or consolidation (Fleming \& Sarker, 1990). There are a number of ways in which the hormones could exert their effects: they could influence processes of learning or of consolidation directly, by acting on the relevant neural substrates of behavior; they could alter attentional mechanisms, in such a way that postpartum animals are simply more alert or attentive to the stimulus situation; they could elevate the animals' overall activity level, increasing the likelihood that animals will contact or engage with the relevant stimuli; they could enhance acuity of the relevant sensory systems, thereby altering the intensity of the perceived stimuli; or they could alter the hedonic value or the salience of the conditioned stimuli. One or another of the parturitional hormones has been shown to produce one or another of these effects. For example, a number of hormones and neurochemicals that are present during the parturitional period have been implicated in learning/consolidation within other nonsocial contexts; these include ACTH, glucocorticoids, oxytocin, the opioids, vasopres- 
sin, and catecholamines (DeWied, 1964; Flood, Jarvick, Bennett, \& Orme, 1976; Harley, 1987; Isaacson, Dunn, Rees, \& Waldock, 1976; Koob, LeMoal, \& Bloom, 1984; Martinez, Schulteis, \& Weinberger, 1991). A number of these are also known to influence social memory specifically (Everitt, 1990; Popik, Wolterink, de Brabander, \& van Ree, 1991). There is, in addition, evidence that arousal and attentional mechanisms can be stimulated by the glucocorticoids (Mason, 1968) and that estradiol stimulates both enhanced motor activity (Quadagno, Shryne, Anderson, \& Gorski, 1972) and enhanced sensory acuity within a number of modalities-particularly the chemosensory (Doty, 1981). Finally, there is evidence within other reproductive contexts that the ovarian steroids increase animals' attraction to sexually related odors (Carr, Loeb, \& Dissinger, 1965).

The extent to which these hormonal effects play a role in social learning in general or in maternal learning specifically is not known (Flood et al., 1976). For maternal learning, however, we have evidence that if virgin animals are administered a hormonal regimen that mimics some of the hormonal characteristics of the parturitional period, they exhibit a more robust maternal experience effect than do those not administered the hormones (Fleming \& Sarker, 1990). Similarly, in sheep, for the ewe to develop an individual recognition of her lamb, she must be exposed to the lamb's olfactory characteristics while she is primed by parturitional hormones (Poindron \& Levy, 1990). Similarly, for both sheep and rats, norepinephrine seems to play a role in the maternal memory learning that takes place (Moffat, Suh, \& Fleming, 1993; Poindron \& Levy, 1990). Unfortunately, we do not know whether the parturitional hormones would promote learning within other functional contexts not involving both the young and maternal behavior.

The absence of a stronger parity effect in these experiments may reflect the fact that females are in general extremely adept at these tasks and are considerably better at remembering an experience than we had expected. With exposure intervals as short as $5 \mathrm{~min}$, animals exhibited a conditioned food preference over a week later (Experiment 1 ); moreover, after brief 30 -min encounters with a juvenile, animals were able to show individual recognition up to 5 days later, despite the fact that the juvenile was somewhat older at test and derived from the same inbred line (Experiment 2). Had we further reduced the exposure durations and/or lengthened the exposure-test intervals, we might well have found more robust parity differences.

Although these parity differences may contribute to the dam's heightened maternal learning, they probably do not account for it entirely. In fact, it is quite likely that the primary difference between $\mathrm{PP}$ and $\mathrm{V}$ animals consists in their responses to pup stimulation. There is evidence, for example, that dams spend more time than do virgins in investigating nest material belonging to a dam and her pups than in investigating clean nest material (Bauer, 1983; Fleming, Cheung, Myhal, \& Kessler, 1989). This parity difference is probably mediated by the parturitional hormones, since comparisons between virgins administered exogenous progesterone and estradiol and those administered cholesterol mimic these differences (Fleming et al., 1989). Moreover, dams come to prefer odors associated with pups during a maternal experience over those not so associated, suggesting that pups and their odor cues are quite reinforcing to the new mother (Fleming et al., 1994). In the next study in this series, we will explore in some detail the reinforcing value of rat pups to the new mother rat as well as to virgins, with varying amounts of maternal experience and hormonal priming.

\section{REFERENCES}

BAUER, J. H. (1983). Effects of maternal state on the responsiveness to nest odors of hooded rats. Physiology \& Behavior, 30, 229-232.

CARR, W. J., Loeb, L. S., \& Dissinger, M. L. (1965). Responses of rats to sex odors. Journal of Comparative \& Physiological Psychology, 59, 370-377.

DEWIED, T. (1964). Influence of the anterior pituitary in avoidance learning and escape behavior. American Journal of Physiology, 207, 255-259

Doty, R. L. (1981). Olfactory communication in humans. Chemical Senses, 6, 351-376.

EverITT, B. (1990). Sexual motivation: A neural and behavioral analysis of the mechanisms underlying appetitive and copulatory responses of male rats. Neuroscience \& Biobehavioral Reviews, 14, 217-232.

Fleming, A. S., Cheung, U. S., Myhal, N., \& Kessler, Z. (1989). Effects of maternal hormones on 'timidity' and attraction to pup-related odors in females. Physiology \& Behavior, 46, 449-453.

Fleming, A. S., Korsmit, M., \& Deller, M. (1994). Rat pups are potent reinforcers to the maternal animal: Effects of experience, parity, hormones, and dopamine function. Psychobiology, 22, 44-53.

FLEMING, A. S., \& SARKER, J. (1990). Experience-hormone interactions and maternal behavior in rats. Physiology \& Behavior, 47, 1165-1173.

Flood, J. F., JarVick, M. E., Bennett, E. L., \& Orme, A. E. (1976). Effects of ACTH peptide fragments on memory formation. Pharmacology, Biochemistry \& Behavior, 5(Suppl. 1), 41-51.

GALEF, B. G., JR. (1989). Enduring social enhancement of rats' preferences for the palatable and the piquant. Appetite, 13, 81-92.

Galef, B. G., JR., Kennett, D. J., \& Stein, M. (1985). Demonstrator influence on observer diet preference: Effects of simple exposure and the presence of a demonstrator. Animal Learning \& Behavior, $13,25-30$.

Galef, B. G., JR., \& Stein, M. (1985). Demonstrator influence on observer diet preference: Analyses of critical social interactions and olfactory signals. Animal Learning \& Behavior, 13, 31-38.

GaleF, B. G., JR., \& Wigmore, S. W. (1983). Transfer of information concerning distant food: A laboratory investigation of the 'information-centre' hypothesis. Animal Behaviour, 31, 748-758.

HARLEY, C. W. (1987). A role for norepinephrine in arousal, emotion and learning: Limbic modulation by norepinephrine and the kety hypothesis. Progress in Neuro-Psychopharmacology \& Biological Psychiatry, 11, 419-458.

IsaAcson, R. L., Dunn, A. J., Rees, H. E., \& Waldock, B. (1976). ACTH 4-10 and improved use of information in rats. Physiology \& Behavior, 4, 159-162.

Koов, G., LeMoal, M., \& Bloom, F. E. (1984). The role of endorphines in neurobiology, behavior and psychiatric disorder. In C. B. Nemeroff \& A. J. Dunn (Eds.), Peptides, hormones \& behavior (pp. 349-384). New York: SP Medical \& Science Books

Malenfant, S. A., Barry, M., \& Fleming, A. S. (1991). The effects of cycloheximide on olfactory learning and maternal experience effects in postpartum rats. Physiology \& Behavior, 9, 289-294.

Martinez, J. L., Schulteis, G., \& Weinberger, S. (1991). How to increase and decrease the strength of memory traces: The effects of drugs and hormones. In J. L. Martinez \& R. P. Kesner (Eds.), Learn- 
ing and memory: A biological view (pp. 149-198). Orlando, FL: Academic Press.

MASON, J. W. (1968). Review of psychoendocrine research on pituitary adrenocortical system. Psychological Medicine, 30, 576-607.

Moffat, S. D., Suh, E. J., \& Fleming, A. S. (1993). Noradrenergic involvement in the consolidation of maternal experience in postpartum rats. Physiology \& Behavior, 53, 805-811.

Morgan, H. D., Fleming, A. S., \& Stern, J. M. (1991). Somatosensory control of the onset and retention of maternal responsiveness in primiparous Sprague-Dawley rats. Physiology \& Behavior, 51, 541-555.

Myhal, N., \& Fleming, A. S. (1990). MK-801 effects on a learned food preference depends on dosage: Is it disruption of learning or a conditioned aversion? Psychobiology, 18, 428-434.

PoIndron, P., \& LEVy, F. (1990). Physiological, sensory and experiential determinants of maternal behavior in sheep. In N. A. Krasnegor \& R. S. Bridges (Eds.), Mammalian parenting: Biochemical, neurobiological and behavioral determinants (pp. 133-156). New York: Oxford University Press.
Popik, P., Wolterink, G., De Brabander, H., \& VAN ReE, J. (1991). Neuropeptides related to $\{$ Arg8 $\}$ vasopressin facilitates social recognition in rats. Physiology \& Behavior, 49, 1031-1035.

Quadagno, D. M., Shryne, J., Anderson, A., \& Gorski, R. A. (1972). Influences of gonadal hormones on social, sexual, emergence and open-field behavior in the rat. (Rattus norvegicus). Animal Behaviour, 20, 732-740.

Sawyer, T. F., Hengehold, A. K., \& Perez, W. A. (1984). Chemosensory and hormonal mediation of social memory in rats. Behavioral Neuroscience, 98, 908-913.

Thor, D. H., \& Holloway, W. R. (1982). Social memory of the male laboratory rat. Journal of Comparative \& Physiological Psychology, 96, 1000-1006.

WINOCUR, G. (1990). Anterograde and retrograde amnesia in rats with dorsal hippocampal or dorsomedial thalamic lesions. Behavioral Brain Research, 38, 145-154.

(Manuscript received March 18, 1993; revision accepted for publication August 19, 1993.) 